\title{
A FILLED FUNCTION METHOD FOR BOX CONSTRAINED NONLINEAR INTEGER PROGRAMMING
}

\author{
YouJiang Lin AND YONGJIAN YANG
}

\begin{abstract}
A new filled function method is presented in this paper to solve box-constrained nonlinear integer programming problems. It is shown that for a given non-global local minimizer, a better local minimizer can be obtained by local search starting from an improved initial point which is obtained by locally solving a box-constrained integer programming problem. Several illustrative numerical examples are reported to show the efficiency of the present method.
\end{abstract}

\section{Introduction}

In [13], Zhu showed that over unbounded domain, the integer programming is undecidable; i.e., there cannot be any algorithm for the problem. So we consider the following box-constrained discrete global optimization problem:

$$
\min \left\{f(x): x \in X \subset \mathbb{Z}^{n}\right\},
$$

where $f: \mathbb{Z}^{n} \rightarrow \mathbb{R}$, and $\mathbb{Z}^{n}$ is the set of integer points in $\mathbb{R}^{n}$, and $X$ is box; i.e., $X=\left\{x \in \mathbb{Z}^{n}: a \leq x \leq b, a, b \in \mathbb{Z}^{n}\right\}$.

Like the continuous global optimization problems, the existence of multiple local minima of a general nonconvex objective function makes discrete global optimization a great challenge. For continuous global optimization problems, many deterministic methods (see [1], [7], [8], [10]) have been proposed to search for a globally optimal solution of a function of several variables. The filled function algorithm (see [2], [4]) is an effective and practical method among determinate algorithms. The primary filled function was proposed by Ge in paper [2]. The definition of the filled function is as follows:

Definition 1.1. Assume that $x_{1}^{*}$ is a current minimizer of $f(x)$. A continuous function $P(x)$ is said to be a filled function of $f(x)$ at $x_{1}^{*}$ if it satisfies the following properties:

Received March 8, 2010; Revised July 26, 2010.

2010 Mathematics Subject Classification. 52A10, 52A40.

Key words and phrases. global optimization, filled function method, nonlinear integer programming, local minimizer, global minimizer.

The authors would like to acknowledge the support from the National Natural Science Foundation of China (10971128), Shanghai Leading Academic Discipline Project (S30104). 
(1) $x_{1}^{*}$ is a maximizer of $P(x)$ and the whole basin $B_{1}^{*}$ of $f(x)$ at $x_{1}^{*}$ becomes a part of a hill of $P(x)$;

(2) $P(x)$ has no minimizers or saddle points in any higher basin of $f(x)$ than $B_{1}^{*}$;

(3) If $f(x)$ has a lower basin than $B_{1}^{*}$, then there is a point $x^{\prime}$ in such a basin that minimizes $P(x)$ on the line through $x$ and $x_{1}^{*}$.

For the definitions of basin and hill, refer to Ge (1990), paper [2].

The filled function given at $x_{1}^{*}$ in the paper [2] has the following form:

$$
P\left(x, x_{1}^{*}, r, \rho\right)=\frac{1}{r+f(x)} \exp \left(-\frac{\left\|x-x_{1}^{*}\right\|^{2}}{\rho^{2}}\right),
$$

where the parameters $r$ and $\rho$ need to be chosen appropriately.

The main idea of the filled function method is to construct an auxiliary function called filled function via the current local minimizer of the original optimization problem, with the property that the current local minimizer is a local maximizer of the constructed filled function and a better initial point of the primal optimization problem can be obtained by minimizing the constructed filled function locally. By the same idea as that of solving continuous global optimization problems, We try to solve discrete global optimization problem (1.1).

In [3], Ge adapted the filled function method by Ge [2] for continuous global optimization to the discrete case. However, the filled function algorithm described in the paper [2] still has some unexpected features:

(i) The efficiency of the filled function algorithm strongly depend on two parameters $r$ and $q$. They are not so easy to be adjusted to make them satisfy the needed conditions;

(ii) The filled function includes exponential terms. If the value of $1 / \rho$ becomes large as iterations proceed, as required to preserve the filling property, numerical illness may result in failure of computation;

(iii) The termination criteria is not good because it requires a large amount of computation before a global minimizer has been found.

In this paper, we provide a new definition of the discrete filled function, and a discrete filled function satisfying the definition is presented. An algorithm is developed from the new filled function. The new filled function algorithm overcomes the disadvantages mentioned above in a certain extent. Specifically, it has the following several advantages:

(i) The new filled function includes neither exponential terms nor logarithmic terms. Elementary function $\phi(t)=$ arctant is used in the filled function, which possesses many good properties and is efficient in numerical implementations.

(ii) The parameters $q$ and $r$ in the new filled function are easier to be appropriately chosen than those of the original filled function (1.2).

(iii) The new algorithm has a simple termination criteria and the computational results show that this algorithm is more efficient than the original algorithm. 
The rest of this paper is organized as follows. In Section 2, we first recall some definitions in discrete analysis and discrete optimization. In Section 3 , a definition of discrete filled function is given, a discrete filled function is presented, and we investigate its properties. An algorithm is developed and numerical experiments are presented in Section 4. Finally, some conclusions are drawn in Section 5.

\section{Preliminary}

First, we recall some definitions in discrete analysis and discrete optimization (see [11], [12]).

Definition 2.1. A sequence $\left\{x^{i}\right\}_{i=-1}^{u}$ is called a discrete path in $X$ between two distinct point $x^{*}$ and $x^{* *}$ in $X$ if $x^{-1}=x^{*}, x^{u}=x^{* *}, x^{i} \in X$ for all $i$; $x^{i} \neq x^{j}$ for $i \neq j$; and $\left\|x^{0}-x^{*}\right\|=\left\|x^{i+1}-x^{i}\right\|=\left\|x^{* *}-x^{u-1}\right\|=1$ for all $i$. If such a discrete path exists, then $x^{*}$ and $x^{* *}$ are said to be pathwise connected in $X$. Furthermore, if every two distinct points in $X$ are pathwise connected in $X$, then $X$ is called a pathwise connected set.

Definition 2.2. The set of all axial directions in $\mathbb{Z}^{n}$ is defined by $D=\left\{ \pm e_{i}\right.$ : $i=1,2, \ldots, n\}$, where $e_{i}$ is the $i$ th unit vector; The set of all feasible directions at $x \in X$ is defined by $D_{x}=\{d \in D: x+d \in X\}$, where $D$ is the set of axial directions.

Definition 2.3. For any $x \in \mathbb{Z}^{n}$, the discrete neighborhood of $x$ is defined by $N(x)=\left\{x, x \pm e_{i}: i=1,2, \ldots, n\right\}$; The discrete interior of $X$ is defined by int $X=\{x \in X: N(x) \subseteq X\}$. While, the discrete boundary of $X$ is denoted by $\partial X=X /$ int $X$.

Definition 2.4. A point $x^{*} \in X$ is called a discrete local minimizer of $f$ over $X$ if $f\left(x^{*}\right) \leq f(x)$ for all $x \in X \cap N\left(x^{*}\right)$. If, in addition, $f\left(x^{*}\right)<f(x)$ for all $x \in X \cap N\left(x^{*}\right) /\left\{x^{*}\right\}$, then $x^{*}$ is called a strict discrete local minimizer of over $X$; A point $x^{*} \in X$ is called a discrete global minimizer of $f$ over $X$ if $f\left(x^{*}\right) \leq f(x)$ for all $x \in X$. If, in addition, $f\left(x^{*}\right)<f(x)$ for all $x \in X$, then $x^{*}$ is called a strict discrete global minimizer of $f$ over $X$.

Definition 2.5. For any $x \in X, d \in D$ is said to be a discrete descent direction of $f$ at $x$ over $X$ if $x+d \in X$ and $f(x+d)<f(x)$; beside, $d^{*} \in D$ is called a discrete steepest descent direction of $f$ at $x$ over $X$ if $f\left(x+d^{*}\right) \leq f(x+d)$ for all $d \in D^{*}$, where $D^{*}$ is the set of all descent direction of $f$ at $x$ over $X$.

Algorithm 2.6. (Discrete steepest descent method).

1. Start from the initial point $x \in X$.

2. If $x$ is a local minimizer of $f$ over $X$, then stop. Otherwise, a discrete steepest descent direction $d^{*}$ of $f$ at $x$ over $X$ can be found.

3. Let $x:=x+\lambda d^{*}$, where $\lambda \in Z_{+}$is the step length such that $f$ has maximum reduction in direction $d^{*}$, and go to Step 2 . 
Algorithm 2.7. (Modified discrete descent method).

1. Start from the initial point $x \in X$.

2. If $x$ is a local minimizer of $f$ over $X$, then stop. Otherwise, let

$$
d^{*}=\operatorname{argmin}\left\{f\left(x+d_{i}\right): d_{i} \in D_{x}, f\left(x+d_{i}\right)<f(x)\right\},
$$

where $D_{x}$ denotes the set of feasible directions at $x$.

3. Let $x:=x+d^{*}$, and go to Step 2 .

Obviously, by Algorithms 2.6 and 2.7, we can only find a discrete local minimizer.

Finally, for the discrete global optimization problem (1.1), we make the following assumptions in this paper:

Assumption 2.8. $X \subseteq \mathbb{Z}^{n}$ is a bounded set which contains more than one point. This implies that there exists a constant $K>0$ such that

$$
1 \leq K=\max _{x, y \in X}\|x-y\| \leq \infty
$$

where $\|\cdot\|$ is the usual Euclidean norm.

Assumption 2.9. $f: \bigcup_{x \in X} N(x) \rightarrow \mathbb{R}$ satisfies the following Lipschiz condition for every $x, y \in \bigcup_{x \in X} N(x)$ :

$$
|f(x)-f(y)| \leq L\|x-y\|,
$$

where $0<L<\infty$ is a constant, $N(x)$ is the discrete neighborhood of $x$.

\section{A filled function and its properties}

In this section, we propose a filled function of $f(x)$ at a current local minimizer and discuss its properties. Let

$$
\begin{aligned}
& S_{1}=\left\{x: \mid f(x) \geq f\left(x_{1}^{*}\right), x \in X \backslash\left\{x_{1}^{*}\right\}\right\}, \\
& S_{2}=\left\{x \mid f(x)<f\left(x_{1}^{*}\right), x \in X\right\} .
\end{aligned}
$$

Definition 3.1. $P\left(x, x_{1}^{*}\right)$ is called a discrete filled function of $f(x)$ at a discrete local minimizer $x_{1}^{*}$ if $P\left(x, x_{1}^{*}\right)$ has the following properties:

(1) $x_{1}^{*}$ is a strict discrete local maximizer of $P\left(x, x_{1}^{*}\right)$ on $X$;

(2) $P\left(x, x_{1}^{*}\right)$ has no discrete local minimizers in the region $S_{1}$;

(3) If $x_{1}^{*}$ is not a discrete global minimizer of $f(x)$, then $P\left(x, x_{1}^{*}\right)$ does have a discrete minimizer in the region $S_{2}$.

Now, we give a discrete filled function for problem (1.1) at a local minimizer $x_{1}^{*}$ as follows:

$$
F\left(x, x_{1}^{*}, q, r\right)=\frac{1}{q+\left\|x-x_{1}^{*}\right\|} \phi_{q}\left(\max \left\{f(x)-f\left(x_{1}^{*}\right)+r, 0\right\}\right),
$$

where

$$
\phi_{q}(t)= \begin{cases}\arctan \left(-\frac{q}{t}\right)+\frac{\pi}{2}, & \text { if } t \neq 0, \\ 0, & \text { if } t=0 .\end{cases}
$$




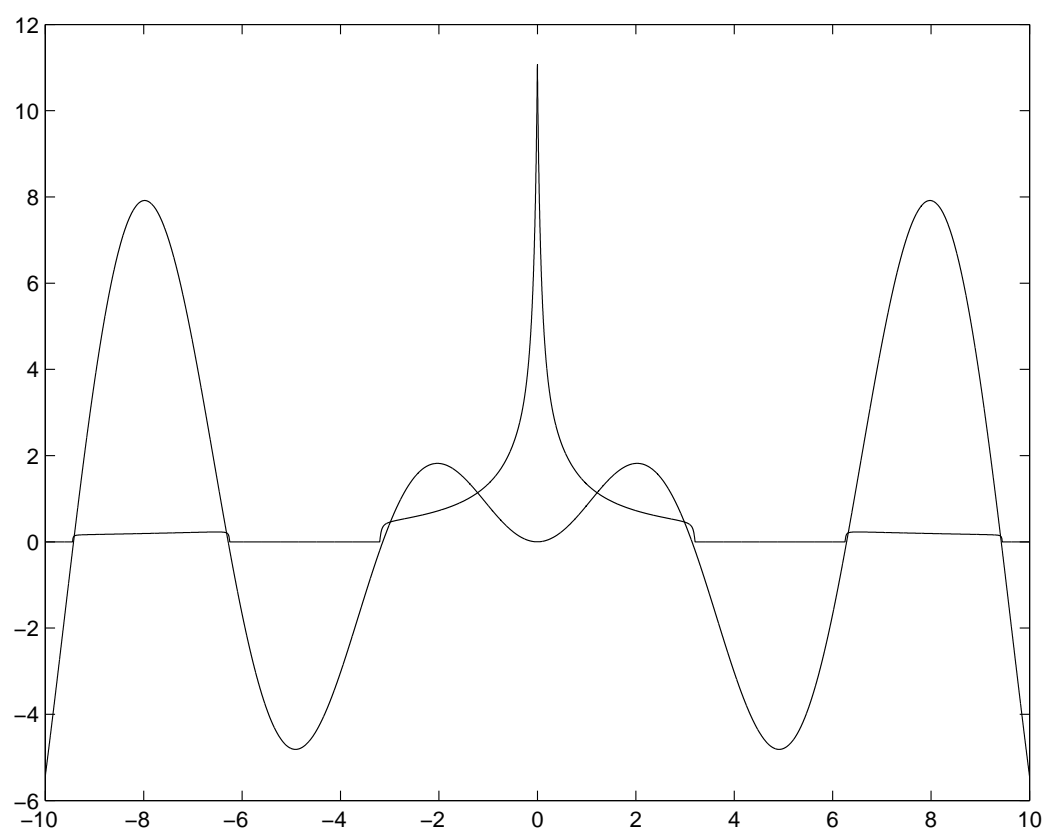

FiguRE 1. $f(x)=x \sin x$ and $F\left(x, x_{1}^{*}, q, r\right)$, where $q=0.1$, $r=0.2$

$q>0$ and $r$ satisfies

$$
0<r<\max _{\substack{x^{*}, x_{1}^{*} \in L(P) \\ f\left(x^{*}\right)<f\left(x_{1}^{*}\right)}}\left(f\left(x_{1}^{*}\right)-f\left(x^{*}\right)\right)
$$

where $L(P)$ stand for the set of discrete local minimizers of $f(x)$. A simple example is given in Figure 1.

Next we will show that the function $F\left(x, x_{1}^{*}, q, r\right)$ is a discrete filled function satisfying Definition 3.1 under certain conditions on the parameters $q$ and $r$.

Theorem 3.2. Suppose that $X$ holds Assumption 2.8. Further suppose that $x_{1}^{*}$ is a discrete local minimizer of $f(x)$. For any $r>0$, when $q>0$ is satisfactorily small, $x_{1}^{*}$ is a strict discrete local maximizer of $F\left(x, x_{1}^{*}, q, r\right)$.

Proof. Since $x_{1}^{*}$ is a discrete local minimizer of $f(x)$ for any $x \in N\left(x_{1}^{*}\right) \cap X$, $f(x) \geq f\left(x_{1}^{*}\right)$ and $\left\|x-x_{1}^{*}\right\|=1$. Hence, we have

$$
F\left(x, x_{1}^{*}, q, r\right)=\frac{1}{q+1}\left(\arctan \left(-\frac{q}{f(x)-f\left(x_{1}^{*}\right)+r}\right)+\frac{\pi}{2}\right)
$$

and

$$
F\left(x_{1}^{*}, x_{1}^{*}, q, r\right)=\frac{1}{q}\left(\arctan \left(-\frac{q}{r}\right)+\frac{\pi}{2}\right),
$$


when $x \geq y, \arctan (x)-\arctan (y) \leq x-y$; let $q<r$, we have

$$
\begin{aligned}
& F\left(x, x_{1}^{*}, q, r\right)-F\left(x_{1}^{*}, x_{1}^{*}, q, r\right) \\
= & \frac{1}{q+1}\left(\arctan \left(-\frac{q}{f(x)-f\left(x_{1}^{*}\right)+r}\right)+\frac{\pi}{2}\right)-\frac{1}{q}\left(\arctan \left(-\frac{q}{r}\right)+\frac{\pi}{2}\right) \\
= & \frac{1}{q+1} \arctan \left(-\frac{q}{f(x)-f\left(x_{1}^{*}\right)+r}\right)-\frac{1}{q} \arctan \left(-\frac{q}{r}\right)+\frac{-1}{q(q+1)} \frac{\pi}{2} \\
= & \frac{1}{q+1} \times\left(\arctan \left(\frac{q}{r}\right)-\arctan \left(\frac{q}{f(x)-f\left(x_{1}^{*}\right)+r}\right)\right) \\
& +\frac{1}{q \times(q+1)}\left(\arctan \left(\frac{q}{r}\right)-\frac{\pi}{2}\right) \\
< & \frac{1}{q \times(q+1)} \times\left(q \times\left(\frac{q}{r}-\frac{q}{f(x)-f\left(x_{1}^{*}\right)+r}\right)+\left(\arctan 1-\frac{\pi}{2}\right)\right) \\
= & \frac{1}{q \times(q+1)} \times\left(q^{2} \times \frac{f(x)-f\left(x_{1}^{*}\right)}{r \times\left(f(x)-f\left(x_{1}^{*}\right)+r\right)}-\frac{\pi}{4}\right) \\
\leq & \frac{1}{q \times(q+1)} \times\left(q^{2} \times \frac{L\left\|x-x_{1}^{*}\right\|}{r^{2}}-\frac{\pi}{4}\right) \\
= & \frac{1}{q \times(q+1)} \times\left(q^{2} \times \frac{L}{r^{2}}-\frac{\pi}{4}\right) \\
\leq & \frac{1}{q \times(q+1)} \times\left(q \times \frac{L}{r}-\frac{\pi}{4}\right) .
\end{aligned}
$$

Therefore, when $0<q<\min \left\{r, \frac{\pi r}{4 L}\right\}$, we have $F\left(x, x_{1}^{*}, q, r\right)-F\left(x_{1}^{*}, x_{1}^{*}, q, r\right)<$ 0 for any $x \in N\left(x_{1}^{*}\right) \cap X, x_{1}^{*}$ is a strict discrete local maximizer of $F\left(x, x_{1}^{*}, q, r\right)$.

Lemma 3.3. For every $x, x^{*} \in X$, if there exits $i \in\{1,2, \ldots, n\}$ such that $x \pm e_{i} \in X$, then there exists $d \in D$ such that

$$
\left\|x+d-x^{*}\right\|>\left\|x-x^{*}\right\| .
$$

Proof. If there is an $i \in\{1,2, \ldots, n\}$ such that $x \pm e_{i} \in X$, then either $\| x+$ $e_{i}-x^{*}\|>\| x-x^{*} \|$ or $\left\|x-e_{i}-x^{*}\right\|>\left\|x-x^{*}\right\|$, therefore we let $d=e_{i}$ or $d=-e_{i}$, this completes the proof.

Theorem 3.4. Suppose that Assumptions 2.8-2.9 are satisfied. If $x_{1}^{*}$ is a discrete local minimizer of $f(x)$, then the function $F\left(x, x_{1}^{*}, q, r\right)$ has no discrete local minimizers in the region $S_{1}=\left\{x \mid f(x) \geq f\left(x_{1}^{*}\right), x \in X /\left\{x_{1}^{*}\right\}\right\}$ when $r>0$ and $q>0$ are satisfactorily small.

Proof. Let $\tilde{X}=\bigcup_{x \in X} N(x)$, obviously, $\tilde{X}$ holds Assumptions 2.8-2.9, and we have $S_{1} \subseteq X \subseteq$ int $\tilde{X}$. For every $x \in S_{1}$, by Lemma 3.3, there must exists a direction $d \in D$ such that $x+d \in X$ and

$$
\left\|x+d-x_{1}^{*}\right\|>\left\|x-x_{1}^{*}\right\| .
$$


Consider the following two cases:

(1) $f(x+d) \geq f\left(x_{1}^{*}\right)$ : Since $f(x+d) \geq f\left(x_{1}^{*}\right)$, we have

$$
\begin{aligned}
& F\left(x+d, x_{1}^{*}, q, r\right)-F\left(x, x_{1}^{*}, q, r\right) \\
= & \frac{1}{q+\left\|x+d-x_{1}^{*}\right\|}\left(\arctan \left(-\frac{q}{f(x+d)-f\left(x_{1}^{*}\right)+r}\right)+\frac{\pi}{2}\right) \\
& -\frac{1}{q+\left\|x-x_{1}^{*}\right\|}\left(\arctan \left(-\frac{q}{f(x)-f\left(x_{1}^{*}\right)+r}\right)+\frac{\pi}{2}\right) \\
= & \frac{1}{q+\left\|x+d-x_{1}^{*}\right\|} \arctan \left(-\frac{q}{f(x+d)-f\left(x_{1}^{*}\right)+r}\right) \\
& -\frac{1}{q+\left\|x-x_{1}^{*}\right\|} \arctan \left(-\frac{\|}{f(x)-f\left(x_{1}^{*}\right)+r}\right) \\
& +\frac{\pi x-x_{1}^{*}\|-\| x+d-x_{1}^{*} \|}{\left(q+\left\|x+d-x_{1}^{*}\right\|\right)\left(q+\left\|x-x_{1}^{*}\right\|\right)} \frac{\pi}{2} \\
= & \frac{1}{q+\left\|x+d-x_{1}^{*}\right\|} \\
& \times\left(\arctan \left(\frac{\left\|x+d-x_{1}^{*}\right\|-\left\|x-x_{1}^{*}\right\|}{f(x)-f\left(x_{1}^{*}\right)+r}\right)-\arctan \left(\frac{q}{f(x+d)-f\left(x_{1}^{*}\right)+r}\right)\right) \\
& +\frac{\left.\arctan \left(\frac{q}{f(x)-f\left(x_{1}^{*}\right)+r}\right)-\frac{\pi}{2}\right) .}{\left(q+\left\|x+d-x_{1}^{*}\right\|\right)\left(q+\left\|x-x_{1}^{*}\right\|\right)} .
\end{aligned}
$$

If $f(x)>f(x+d)$, then

$$
\arctan \left(\frac{q}{f(x)-f\left(x_{1}^{*}\right)+r}\right)-\arctan \left(\frac{q}{f(x+d)-f\left(x_{1}^{*}\right)+r}\right)<0
$$

therefore when $q<r$, we have $\arctan \left(\frac{q}{f(x)-f\left(x_{1}^{*}\right)+r}\right)-\frac{\pi}{2}<0$, hence

$$
F\left(x+d, x_{1}^{*}, q, r\right)-F\left(x, x_{1}^{*}, q, r\right)<0 .
$$

If $f(x) \leq f(x+d)$ for any $x, y \in R$, when $x \leq y$, we have $\arctan y-\arctan x \leq$ $y-x$, let $q<r$ and $q<1$, we have

$$
\begin{aligned}
& F\left(x+d, x_{1}^{*}, q, r\right)-F\left(x, x_{1}^{*}, q, r\right) \\
\leq & \frac{1}{q+\left\|x+d-x_{1}^{*}\right\|} \times\left(\frac{q}{f(x)-f\left(x_{1}^{*}\right)+r}-\frac{q}{f(x+d)-f\left(x_{1}^{*}\right)+r}\right) \\
& +\frac{\left\|x+d-x_{1}^{*}\right\|-\left\|x-x_{1}^{*}\right\|}{\left(q+\left\|x+d-x_{1}^{*}\right\|\right)\left(q+\left\|x-x_{1}^{*}\right\|\right)}\left(\arctan 1-\frac{\pi}{2}\right) \\
\leq & \frac{q}{q+\left\|x+d-x_{1}^{*}\right\|} \times\left(\frac{f(x+d)-f(x)}{\left(f(x)-f\left(x_{1}^{*}\right)+r\right)\left(f(x+d)-f\left(x_{1}^{*}\right)+r\right)}\right) \\
& +\frac{\left\|x+d-x_{1}^{*}\right\|-\left\|x-x_{1}^{*}\right\|}{\left(q+\left\|x+d-x_{1}^{*}\right\|\right)\left(2\left\|x-x_{1}^{*}\right\|\right)}\left(\arctan 1-\frac{\pi}{2}\right) \\
\leq & \frac{1}{\left(q+\left\|x+d-x_{1}^{*}\right\|\right)\left(2\left\|x-x_{1}^{*}\right\|\right)}
\end{aligned}
$$




$$
\times\left(\frac{2 q L\|d\|\left\|x-x_{1}^{*}\right\|}{r^{2}}-\frac{\pi}{4}\left(\left\|x+d-x_{1}^{*}\right\|-\left\|x-x_{1}^{*}\right\|\right)\right) .
$$

Hence, for any given $x \in S_{1}$ and $d$, when $q$ is satisfactorily small that

$$
q<\frac{\pi r^{2}\left(\left\|x+d-x_{1}^{*}\right\|-\left\|x-x_{1}^{*}\right\|\right)}{8 L\left\|x-x_{1}^{*}\right\|},
$$

we have

$$
F\left(x+d, x_{1}^{*}, q, r\right)<F\left(x, x_{1}^{*}, q, r\right) .
$$

(2) $f(x+d)<f\left(x_{1}^{*}\right)$ : In this case, it is clear that $f(x+d)<f(x)$, since function $f(t)=\arctan \left(-\frac{q}{t}\right)+\frac{\pi}{2}$ is increasing about $t$, hence, we have

$$
F\left(x+d, x_{1}^{*}, q, r\right)<F\left(x, x_{1}^{*}, q, r\right) .
$$

The above two cases imply that any $x \in S_{1}$ is not the discrete local minimizer of $F\left(x, x_{1}^{*}, q, r\right)$ when $q$ is satisfactorily small.

Theorem 3.5. If $x_{1}^{*}$ is not a discrete global minimizer of $f(x)$ in $X$, then there exists a discrete minimizer ${\overline{x_{1}}}^{*}$ of $F\left(x, x_{1}^{*}, q, r\right)$ in the region $S_{2}=\{x \mid f(x)<$ $\left.f\left(x_{1}^{*}\right), x \in X\right\}$.

Proof. Since $x_{1}^{*}$ is not a discrete global minimizer and $F\left(x, x_{1}^{*}, q, r\right) \geq 0$, there exist a point $\overline{x_{1}}{ }^{*} \in S_{2}$ and $r$ such that $f\left(\overline{x_{1}}{ }^{*}\right)<f\left(x_{1}^{*}\right)-r$. Hence, $F\left(\overline{x_{1}}{ }^{*}, x_{1}^{*}, q, r\right)$ $=0$, it implies that $\bar{x}_{1}^{*} \in S_{2}$ is a discrete minimizer of $F\left(x, x_{1}^{*}, q, r\right)$.

Theorem 3.2, Theorem 3.4, and Theorem 3.5 show that the function $F(x$, $\left.x_{1}^{*}, q, r\right)$ at point $x_{1}^{*}$ is a discrete filled function satisfying Definition 3.1 with satisfactorily small $q$ and $r$. The following theorems further show that the proposed filled function has some good properties which classical functions have.

Theorem 3.6. Suppose that Assumption 2.9 is satisfied. If $x_{1}, x_{2} \in X$ and satisfy the following conditions:

(1) $f\left(x_{1}\right) \geq f\left(x_{1}^{*}\right)$ and $f\left(x_{2}\right) \geq f\left(x_{1}^{*}\right)$,

(2) $\left\|x_{2}-x_{1}^{*}\right\|>\left\|x_{1}-x_{1}^{*}\right\|$.

Then, when $r>0$ and $q>0$ are satisfactorily small,

$$
F\left(x_{2}, x_{1}^{*}, q, r\right)<F\left(x_{1}, x_{1}^{*}, q, r\right) .
$$

Proof. Consider the following two cases:

(1) If $f\left(x_{1}^{*}\right) \leq f\left(x_{2}\right) \leq f\left(x_{1}\right)$, then it is obvious that the result follows.

(2) If $f\left(x_{1}^{*}\right) \leq f\left(x_{1}\right)<f\left(x_{2}\right)$, the result also holds (see the proof process in Theorem 3.4).

Theorem 3.7. If $x_{1}, x_{2} \in X$ and satisfy the following conditions:

(1) $\left\|x_{2}-x^{*}\right\|>\left\|x_{1}-x^{*}\right\|$,

(2) $f\left(x_{1}\right) \geq f\left(x_{1}^{*}\right)>f\left(x_{2}\right)$, and $f\left(x_{2}\right)-f\left(x_{1}^{*}\right)+r>0$.

Then, we have $F\left(x_{2}, x_{1}^{*}, r, q\right)<F\left(x_{1}, x_{1}^{*}, r, q\right)$. 
Proof. By Conditions 1 and 2, we have

and

$$
\frac{1}{q+\left\|x_{2}-x_{1}^{*}\right\|}<\frac{1}{q+\left\|x_{1}-x_{1}^{*}\right\|}
$$

$$
0<f\left(x_{2}\right)-f\left(x_{1}^{*}\right)+r<f\left(x_{1}\right)-f\left(x_{1}^{*}\right)+r .
$$

Hence $F\left(x_{2}, x_{1}^{*}, r, q\right)<F\left(x_{1}, x_{1}^{*}, r, q\right)$.

Now we make some remarks. Firstly, in the phase of minimizing the new discrete filled function, Theorems 3.6 and 3.7 guarantee that the current discrete local minimizer $x_{1}^{*}$ of the objective function is escaped and the minimum of the new discrete filled function will be always achieved at a point where the objective function value is less than the current discrete minimum. Secondly, the parameters $q$ and $r$ are easier to be appropriately chosen. In the next section, a new discrete filled function algorithm is given.

\section{Algorithm and numerical results}

Based on the theoretical results in the previous section, a global optimization algorithm over $X$ is proposed as follows:

\section{Algorithm}

Initialization:

(1) Choose any $x_{0} \in X$ as an initial point.

(2) Let $\varepsilon=10^{-5}$ and $q_{0}=0.01$.

(3) Let $D_{0}=\left\{ \pm e_{i}: i=1,2, \ldots, n\right\}$.

Main Program:

(1) Starting from initial point $x_{0}$, minimize $f(x)(x \in X)$ by the discrete steepest descent method (see Algorithm 2.1), we can obtain the discrete local minimizer $x_{1}^{*}$.

Let $r=1, q=q_{0}$ and $D=D_{0}$.

(2) Construct the discrete filled function:

$$
F\left(x, x_{1}^{*}, q, r\right)=\frac{1}{q+\left\|x-x^{*}\right\|} \phi_{q}\left(\max \left\{f(x)-f\left(x_{1}^{*}\right)+r, 0\right\}\right),
$$

where

$$
\phi_{q}(t)= \begin{cases}\arctan \left(-\frac{q}{t}\right)+\frac{\pi}{2}, & \text { if } t \neq 0, \\ 0, & \text { if } t=0 .\end{cases}
$$

(3) If $r \leq \varepsilon$, then terminate the iteration, the $x_{k}^{*}$ is the global minimizer of $f(x)$, otherwise, the next step.

(4) If $D \neq \emptyset$, then goto (6), otherwise the next step.

(5) If $q<\varepsilon \times 10^{-2}$, then let $r=r / 10, q=q_{0} / 10$ and $D=D_{0}$, goto (2); otherwise let $q=q / 10$, goto $(2)$.

(6) Take a direction $d \in D$, and $D \leftarrow D /\{d\}$, turn to Inner Loop. 
Inner Loop:

(1) $k=0$.

(2) Let $y_{k}=x_{1}^{*}+d$.

(3) Minimize $F\left(x, x_{1}^{*}, q, r\right)$, starting from the point $y_{k}$, by implementing the modified discrete descent method (see Algorithm 2.2). $y_{k+1}$ denotes the next iterative point.

(4) If $y_{k+1} \notin X$, then return Main Program (4), otherwise next step.

(5) If $f\left(y_{k+1}\right) \leq f\left(x_{1}^{*}\right)$, then let $x_{0}=y_{m+1}$ and return Main program (1), otherwise let $k=k+1$ and goto Inner Loop (3).

In the following part, several test problems are given and results of the algorithm in solving these problems are reported. Through out the tests, we use the modified discrete descent method as shown in Algorithm 2.7 to perform local searches, in the initialization of the algorithm we let $q=0.01$ and $r=1$. The algorithm in Fortran 95 is successfully used to find the global minimizers of these test problems.

The main iterative results are summarized in tables for each function. The symbols used are shown as follows:

$k \quad:$ The iteration number in finding the $k$ th local minimizer.

$x_{k}^{0}$ or $y_{k}^{0} \quad:$ The $k$ th initial point.

$f\left(x_{k}^{0}\right)$ or $f\left(y_{k}^{0}\right)$ : The function value of the $k$ th initial point.

$x_{k}^{*}$ or $y_{k}^{*} \quad:$ The $k$ th local minimizer.

$f\left(x_{k}^{*}\right)$ or $f\left(y_{k}^{*}\right)$ : The function value of the $k$ th local minimizer.

\section{Problem 4.1.}

$$
\begin{aligned}
& \min \quad f(x)= 100\left(x_{2}-x_{1}^{2}\right)^{2}+\left(1-x_{1}\right)^{2}+90\left(x_{4}-x_{3}^{2}\right)^{2}+\left(1-x_{3}\right)^{2}, \\
&+10.1\left[\left(x_{2}-1\right)^{2}+\left(x_{4}-1\right)^{2}\right]+19.8\left(x_{2}-1\right)\left(x_{4}-1\right), \\
& \text { s.t. } \quad 10 \leq x_{i} \leq 10, i=1,2,3,4 .
\end{aligned}
$$

This problem is a discrete counterpart of the problem 38 in [6]. It is a box constrained nonlinear integer programming problem. It has $21^{4} \approx 1.94 \times 10^{5}$ feasible points where 41 of them are discrete local minimizers but only one of those discrete local minimizers is the discrete global minimum solution: $x_{\text {global }}^{*}=(1,1,1,1)$ with $f\left(x_{\text {global }}^{*}\right)=0$. Let $x_{1}^{0}=(9,6,5,6)$ and $x_{1}^{0}=$ $(9,-9,-9,9)$, a summary of the computational results are displayed in Tables 1 and 2 .

\section{Problem 4.2.}

$$
\begin{array}{cl}
\min & f(x)=g(x) h(x), \\
\text { s.t. } & x_{i}=0.001 y_{i}, \quad-2000<y_{i}<2000, \quad i=1,2,
\end{array}
$$

where $y_{i}(i=1,2)$ is integer, and

$$
\begin{array}{r}
g(x)=1+\left(x_{1}+x_{2}+1\right)^{2}\left(19-14 x_{1}+3 x_{1}^{2}-14 x_{2}+6 x_{1} x_{2}+3 x_{2}\right), \\
h(x)=30+\left(2 x_{1}-3 x_{2}\right)^{2}\left(18-32 x_{1}+12 x_{1}^{2}+48 x_{2}-36 x_{1} x_{2}+27 x_{2}^{2}\right) .
\end{array}
$$


TABle 1. Problem 4.1 initial point is $(9,6,5,6)$

\begin{tabular}{l|l|l|l|l}
\hline$k$ & $x_{k}^{0}$ & $f\left(x_{k}^{0}\right)$ & $x_{k}^{*}$ & $f\left(x_{k}^{*}\right)$ \\
\hline 1 & $(9,6,5,6)$ & 596070.0 & $(3,8,3,8)$ & 2158.0000 \\
\hline 2 & $(3,10,2,5)$ & 1887.5000 & $(3,8,2,3)$ & 1007.5000 \\
\hline 3 & $(3,10,1,1)$ & 922.1000 & $(3,8,0,-1)$ & 453.1000 \\
\hline 4 & $(2,5,0,-1)$ & 235.6000 & $(2,4,0,0)$ & 43.6000 \\
\hline 5 & $(1,1,0,0)$ & 11.1000 & $(1,1,1,1)$ & 0.0000 \\
\hline
\end{tabular}

TABLe 2. Problem 4.1 initial point is $(9,-9,-9,9)$

\begin{tabular}{l|l|l|l|l}
\hline$k$ & $x_{k}^{0}$ & $f\left(x_{k}^{0}\right)$ & $x_{k}^{*}$ & $f\left(x_{k}^{*}\right)$ \\
\hline 1 & $(9,-9,-9,9)$ & 1276796.40 & $(3,8,-3,8)$ & 2170.0000 \\
\hline 2 & $(3,10,-2,5)$ & 1895.5000 & $(3,8,-2,3)$ & 1015.5000 \\
\hline 3 & $(3,10,-1,1)$ & 926.1000 & $(3,8,0,-1)$ & 453.1000 \\
\hline 4 & $(2,5,0,-1)$ & 235.6000 & $(2,4,0,0)$ & 43.6000 \\
\hline 5 & $(1,1,0,0)$ & 11.1000 & $(1,1,1,1)$ & 0.0000 \\
\hline
\end{tabular}

TABle 3. Problem 4.2

\begin{tabular}{l|l|l|l|l}
\hline$k$ & $y_{k}^{0}$ & $f\left(y_{k}^{0}\right)$ & $y_{k}^{*}$ & $f\left(y_{k}^{*}\right)$ \\
\hline 1 & $(1000,1000)$ & 1876.000 & $(1280,890)$ & 954.1382 \\
\hline 2 & $(2000,632)$ & 951.2482 & $(1609,71)$ & 92.3425 \\
\hline 3 & $(1379,2000)$ & 19.4895 & $(85,2000)$ & -210795.6253 \\
\hline \hline 1 & $(-1000,-1000)$ & 1890.000 & $(0,-1000)$ & 3.000000 \\
\hline 2 & $(0,1740)$ & -256.5294 & $(85,2000)$ & -210795.6253 \\
\hline \hline 1 & $(2000,2000)$ & 35028.000 & $(1280,890)$ & 954.1382 \\
\hline 2 & $(2000,632)$ & 951.2482 & $(1609,71)$ & 92.3426 \\
\hline 3 & $(1379,2000)$ & 19.4895 & $(85,2000)$ & -210795.6253 \\
\hline \hline 1 & $(-2000,-2000)$ & 20811.9999 & $(0,-1000)$ & 3.000000 \\
\hline 2 & $(0,1740)$ & -256.5294 & $(85,2000)$ & -210795.6253 \\
\hline
\end{tabular}

This problem is a discrete counterpart of the Goldstein and Price's function in [5]. It is a box constrained nonlinear integer programming problem. It has $4001^{2} \approx 1.60 \times 10^{7}$ feasible points. More precisely, it has 207 and 2 discrete local minimizers in the interior and the boundary of box $-2.00 \leq x_{i} \leq 2.00, i=1,2$, respectively. Nevertheless, it has only one discrete global minimum solution: $y_{\text {global }}^{*}=(85,2000)$ with $f\left(y_{\text {global }}^{*}\right)=-210795.6253$. We used four initial points in our experiment: $(1000,1000),(-1000,-1000),(2000,2000),(-2000,-2000)$, a summary of the computational results are displayed in Table 3 .

Problem 4.3 (Beale's function).

$\min f(x)=\left[1.5-x_{1}\left(1-x_{2}\right)\right]^{2}+\left[2.25-x_{1}\left(1-x_{2}^{2}\right)\right]^{2}+\left[2.625-x_{1}\left(1-x_{2}^{3}\right)\right]^{2}$, 
TABle 4. Problem 4.3

\begin{tabular}{l|l|l|l|l}
\hline$k$ & $y_{k}^{0}$ & $f\left(y_{k}^{0}\right)$ & $y_{k}^{*}$ & $f\left(y_{k}^{*}\right)$ \\
\hline 1 & $(3000,5000)$ & 146039.2031 & $(-3,9735)$ & 6.0673 \\
\hline 2 & $(10000,963)$ & 6.0183 & $(3015,504)$ & $3.7589 \times 10^{-5}$ \\
\hline \hline 1 & $(-3000,-5000)$ & 150120.7031 & $(11,-5964)$ & 9.0230 \\
\hline 2 & $(10000,788)$ & 8.9179 & $(3015,504)$ & $3.7589 \times 10^{-5}$ \\
\hline \hline 1 & $(8000,8000)$ & 16992808.2031 & $(-3,9735)$ & 6.0673 \\
\hline 2 & $(10000,963)$ & 6.0183 & $(3015,504)$ & $3.7589 \times 10^{-5}$ \\
\hline \hline 1 & $(-8000,-8000)$ & 17121524.2031 & $(5,-7842)$ & 8.6781 \\
\hline 2 & $(10000,790)$ & 8.6132 & $(3015,504)$ & $3.7589 \times 10^{-5}$ \\
\hline
\end{tabular}

TABLE 5. Problem 4.4

\begin{tabular}{l|l|l|l|l}
\hline$k$ & $y_{k}^{0}$ & $f\left(y_{k}^{0}\right)$ & $y_{k}^{*}$ & $f\left(y_{k}^{*}\right)$ \\
\hline 1 & $(-5000,-5000,-5000,-5000)$ & 3650.0000 & $(-116,12,55,-56)$ & $3.7213 \times 10^{-4}$ \\
\hline 2 & $(-78,8,38,-38)$ & $7.9387 \times 10^{-5}$ & $(-79,8,38,-38)$ & $7.9045 \times 10^{-5}$ \\
\hline 3 & $(11,-1,11,11)$ & $1.2798 \times 10^{-6}$ & $(10,-1,11,11)$ & $2.7985 \times 10^{-7}$ \\
\hline 4 & $(0,0,3,3)$ & $2.1060 \times 10^{-9}$ & $(0,0,1,1)$ & $2.6000 \times 10^{-1}$ \\
\hline \hline 1 & $(5000,5000,5000,5000)$ & 3650.0000 & $(116,-12,56,57)$ & $3.7859 \times 10^{-4}$ \\
\hline 2 & $(78,-8,38,38)$ & $7.9387 \times 10^{-5}$ & $(79,-8,38,38)$ & $7.9045 \times 10^{-5}$ \\
\hline 3 & $(-11,1,-11,-11)$ & $1.2798 \times 10^{-6}$ & $(-10,1,11,-11)$ & $2.7985 \times 10^{-7}$ \\
\hline 4 & $(0,0,3,-3)$ & $2.1060 \times 10^{-9}$ & $(0,0,1,-1)$ & $2.6000 \times 10^{-1} 1$ \\
\hline
\end{tabular}

TABLE 6

\begin{tabular}{|c|c|c|c|c|}
\hline$P N$ & $D N$ & $I N$ & $T I$ & $F N$ \\
\hline 1 & 4 & 5 & 0.2360 & 4953 \\
\hline 2 & 2 & 3 & 0.07073 & 231767 \\
\hline 3 & 2 & 2 & 0.05216 & 224364 \\
\hline 4 & 4 & 4 & 278.2456 & 75801758 \\
\hline 5 & 25 & 2 & 47.49104 & 9503685 \\
\hline 5 & 50 & 2 & 1147.472 & 98451600 \\
\hline 5 & 100 & 2 & 4406.341 & 276667658 \\
\hline 6 & 25 & 2 & 42.67253 & 3672432 \\
\hline 6 & 50 & 2 & 249.2645 & 35436658 \\
\hline 6 & 100 & 2 & 3087.764 & 275437056 \\
\hline
\end{tabular}

s.t. $x_{i}=0.001 y_{i}, \quad-10^{4} \leq y_{i} \leq 10^{4}, \quad i=1,2$,

where $y_{i}(i=1,2)$ is integer.

This problem is discrete counterpart if the problem 203 in [9]. It is a box constrained nonlinear integer programming problem. It has $20001^{2} \approx 4.00 \times 10^{8}$ feasible points and many discrete local minimizers, but it has only one discrete 
TABLE 7. Comparison of the results

\begin{tabular}{ll|llllllll}
\hline & & \multicolumn{2}{l|}{ Ge's algorithm } & & \multicolumn{3}{l}{ New algorithm } \\
\cline { 4 - 9 } PN & $D N$ & IN & TI & $F N$ & $R A$ & IN & TI & $F N$ & $R A$ \\
\hline 1. & 4 & 10 & 73.667 & 70746 & $1.22^{-4}$ & 6 & 38.564 & 46653 & $0.47^{-5}$ \\
& & 32 & 1837.536 & 617564 & $4.73^{-3}$ & 11 & 328.102 & 29576 & $1.78^{-5}$ \\
& & 12 & 657.075 & 112685 & $2.03^{-3}$ & 8 & 375.807 & 52236 & $0.38^{-4}$ \\
& & 35 & 866.267 & 385507 & $6.12^{-4}$ & 18 & 406.542 & 45351 & $3.80^{-6}$ \\
& & 18 & 659.516 & 100841 & $0.24^{-4}$ & 15 & 315.345 & 50055 & $1.67^{-6}$ \\
2. & 2 & 11 & 57.367 & 2352 & $0.10^{-4}$ & 6 & 48.051 & 230 & $3.15^{-6}$ \\
& & 13 & 168.742 & 6590 & $0.67^{-5}$ & 6 & 123.098 & 567 & $5.03^{-7}$ \\
& & 13 & 89.527 & 6350 & $1.25^{-4}$ & 6 & 31.916 & 585 & $0.72^{-6}$ \\
3. & 2 & 6 & 3454.355 & 367459 & $8.07^{-4}$ & 12 & 982.440 & 13422 & $3.90^{-5}$ \\
& & 6 & 7098.621 & 653210 & $9.12^{-4}$ & 10 & 1752.812 & 17983 & $9.92^{-6}$ \\
4. & 4 & 20 & 12873.310 & 5324318 & $3.19^{-3}$ & 16 & 2577.120 & 545390 & $1.10^{-5}$ \\
& & 20 & 5952.546 & 3087641 & $5.00^{-4}$ & 16 & 214.342 & 316634 & $3.00^{-6}$ \\
5. & 25 & 32 & 28750.217 & 8342109 & $1.05^{-6}$ & 18 & 1524.708 & 345297 & $2.13^{-7}$ \\
& & 32 & 6532.097 & 2864310 & $2.09^{-6}$ & 18 & 352.761 & 96531 & $2.20^{-7}$ \\
& & 31 & 6098.732 & 3067812 & $4.56^{-6}$ & 19 & 213.450 & 88206 & $4.12^{-7}$ \\
6. & 25 & 18 & 1105.550 & 368056 & $0.06^{-5}$ & 25 & 428.650 & 30987 & $4.31^{-8}$ \\
& & 18 & 963.271 & 298767 & $5.01^{-5}$ & 25 & 320.710 & 75618 & $3.00^{-8}$ \\
& & 18 & 2544.326 & 802114 & $2.10^{-5}$ & 23 & 484.562 & 8945 & $7.00^{-7}$ \\
& & 18 & 3245.711 & 2354770 & $0.38^{-5}$ & 25 & 145.354 & 234552 & $8.29^{-8}$ \\
\hline
\end{tabular}

global minimum solution $y_{\text {global }}^{*}=(3000,500)$ with $f\left(y_{\text {global }}^{*}\right)=0$. We used four initial points in our experiment: $(3000,5000),(-3000,-5000),(8000,8000)$, $(-8000,-8000)$, a summary of the computational results are displayed in Table 4 .

Problem 4.4 (Powell's singular function).

$$
\begin{array}{cl}
\min & f(x)=\left(x_{1}+10 x_{2}\right)^{2}+5\left(x_{3}-x_{4}\right)^{2}+\left(x_{2}-2 x_{3}\right)^{4}+10\left(x_{1}-x_{4}\right)^{4}, \\
\text { s.t. } & x_{i}=0.001 y_{i}, \quad-10^{4} \leq y_{i} \leq 10^{4}, \quad i=1,2,3,4
\end{array}
$$

where $y_{i}(i=1,2,3,4)$ is integer.

It is a box constrained nonlinear integer programming problem. It has $20001^{4} \approx 1.60 \times 10^{17}$ feasible points and many local minimizers, but it has only one global minimum solution: $y_{\text {global }}^{*}=(0,0,0,0)$ with $f\left(y_{\text {global }}^{*}\right)=0$. We used two initial points in our experiment: $(-5000,-5000,-5000,-5000)$, $(5000,5000,5000,5000)$, a summary of the computational results are displayed in Table 5.

\section{Problem 4.5.}

$$
\begin{array}{ll}
\min & f(x)=\left(x_{1}-1\right)^{2}+5\left(x_{n}-1\right)^{2}+\sum_{i=1}^{n-1}(n-i)\left(x_{i}^{2}-x_{i+1}\right)^{2}, \\
\text { s.t. } & -5 \leq y_{i} \leq 5, \quad i=1,2, \ldots, n .
\end{array}
$$


This problem is a generalization of the problem 282 in [9]. It is a box constrained nonlinear integer programming problem. It has $11^{n}$ feasible points and many local minimizers, but it has only one global minimum solution: $x_{\text {global }}^{*}=$ $(1, \ldots, 1)$ with $f\left(x_{\text {global }}^{*}\right)=0$. For all problem with different size, we used four initial points in our experiment: $(5, \ldots, 5),(-5, \ldots,-5),(-5, \ldots,-5,5, \ldots, 5)$, $(5, \ldots, 5,-5, \ldots,-5)$. For every experiment, the proposed algorithm succeeded in identifying the discrete global minimum. Let $x_{1}^{0}=(5, \ldots, 5)$, for $n=$ $25,50,100$, respectively, the summary of the computational results are displayed in Table 6 .

Problem 4.6 (Rosenbrock's function).

$$
\begin{array}{ll}
\min & f(x)=\sum_{i=1}^{n-1}\left[100\left(x_{i+1}-x_{i}^{2}\right)^{2}+\left(1-x_{i}\right)^{2}\right], \\
\text { s.t. } & -5 \leq y_{i} \leq 5, \quad i=1,2, \ldots, n .
\end{array}
$$

It is a box constrained/unconstrained nonlinear integer programming problem. It has $11^{n}$ feasible points and many local minimizers, but it has only one global minimum solution: $x_{\text {global }}^{*}=(1,1, \ldots, 1)$ with $f\left(x_{\text {global }}^{*}\right)=0$. For all problems with different sizes, we used four initial points in our experiment: $(5, \ldots, 5),(-5, \ldots,-5),(-5, \ldots,-5,5, \ldots, 5),(5, \ldots, 5,-5, \ldots,-5)$. For every experiment, the proposed algorithm succeeded in identifying the discrete global minimum. Let $x_{1}^{0}=(5, \ldots, 5)$, for $n=25,50,100$, respectively, the summary of the computational results are displayed in Table 6 .

In Table 6, we give the experiment results for Problems 4.1-4.6. In Table 7, Problems 4.1-4.6. with 2 to 25 variables are tested and the table shows that in most cases the new filled function algorithm works better than Ge's filled function algorithm. The symbols used in Tables 6-7 are shown as follows:

$P N$ : The $N$ th problem.

$D N$ : The dimension of objective function of a problem.

$I N$ : The number of iteration cycles.

$T I$ : The CPU time in seconds for the algorithm to stop.

$F N$ : The number of objective function evaluations for algorithm to stop.

$R A$ : The ratio of the number of function evaluations to the number of feasible points.

\section{Conclusions}

This paper gives a definition of the filled function for the nonlinear integer programming problem, and presents a new filled function. A filled function algorithm based on this given filled function is designed. The implementation of the algorithm on several test problems is reported with satisfactory numerical results. 
Acknowledgment. The authors are most grateful to the referee for his many excellent suggestions for improving the original manuscript.

\section{References}

[1] B. C. Cetin, J. Barhen, and J. W. Burdick, Terminal repeller unconstrained subenergy tunneling (TRUST) for fast global optimization, J. Optim. Theory Appl. 77 (1993), no. $1,97-126$.

[2] R. P. Ge, A filled function method for finding a global minimizer of a function of several variables, Math. Programming 46 (1990), no. 2, (Ser. A), 191-204.

[3] R. P. Ge and C. B. Huang, A continuous approach to nonlinear integer programming, Appl. Math. Comput. 34 (1989), no. 1, part I, 39-60.

[4] R. P. Ge and Y. F. Qin, The globally convexized filled functions for global optimization, Appl. Math. Comput. 35 (1990), no. 2, part II, 131-158.

[5] A. A. Goldstein and J. F. Price, On descent from local minima, Math. Comp. 25 (1971), $569-574$.

[6] W. Hock and K. Schittkowski, Test Examples for Nonlinear Programming Codes, Springer, NewYork, 1981.

[7] R. Horst, P. M. Pardalos, and N. V. Thoai, Introduction to Global Optimization, second ed., Kluwer Academic Publishers, Dordrecht, 2000.

[8] A. V. Levy and A. Montalvo, The tunneling algorithm for the global minimization of functions, SIAM J. Sci. Statist. Comput. 6 (1985), no. 1, 15-29.

[9] K. Schittkowski, More Test Examples for Nonlinear Programming Codes, Springer, NewYork, 1987.

[10] Y. Yao, Dynamic tunneling algorithm for global optimization, IEEE Trans. Systems Man Cybernet. 19 (1989), no. 5, 1222-1230.

[11] L. S. Zhang, F. Gao, and W. X. Zhu, Nonlinear integer programming and global optimization, J. Comput. Math. 17 (1999), no. 2, 179-190.

[12] W. X. Zhu, An approximate algorithm for nonlinear integer programming, Appl. Math. Comput. 93 (1998), no. 2-3, 183-193.

[13] Unsolvability of some optimization problems, Appl. Math. Comput. 174 (2006), no. 2, 921-926.

YOUJIANG LIN

Department of Mathematics

Shanghai University

Shanghai 200444, P. R. China

E-mail address: linyoujiang@shu.edu.cn

YONGJIAN YANG

Department of Mathematics

Shanghai University

Shanghai 200444, P. R. China

E-mail address: yjyang@mail.shu.edu.cn 